\title{
Analysis on an illusion unexpected occurred on a moving statue leaving in fact but approaching by environmental judgment
}

Youwu He, Zhifang Li, Yishen Qiu, Hui Li

Youwu He, Zhifang Li, Yishen Qiu, Hui Li, "Analysis on an illusion unexpected occurred on a moving statue leaving in fact but approaching by environmental judgment," Proc. SPIE 10452, 14th Conference on Education and Training in Optics and Photonics: ETOP 2017, 1045262 (16 August 2017); doi: $10.1117 / 12.2269904$

SPIE Event: 14th Conference on Education and Training in Optics and Photonics, ETOP 2017, 2017, Hangzhou, China 


\title{
Analysis on an illusion unexpected occurred on a moving
}

\section{statue leaving in fact but approaching by environmental}

\author{
judgement \\ Youwu He, Zhifang $\mathrm{Li}$, Yishen Qiu and $\mathrm{Hui} \mathrm{Li}^{*}$ \\ College of Photonic and Electronic Engineering, Fujian Normal University, \\ Fuzhou 350117, China
}

*Email: hli@fjnu.edu.cn

\begin{abstract}
Earlier this year we visited Sanya, Hainan Province, China. There is a huge statue, the South Sea Avalokitesvara（南海观世音菩萨）, at Sanya Nanshan Buddhism Cultural Tourism Resort. When we were gazing at the statue on a leaving car on gradually rising road, an unexpected visual illusion took place in which the statue seemed running after us. In this presentation, an optical model is developed to explain the illusion occurred on a moving object leaving in fact but approaching by environmental judgement. Such an interesting illusion analysis will play a significant role in having students understood the main principles in geometrical optics.
\end{abstract}

Keywords: visual illusion; optical model; geometrical optics;

\section{Introduction}

Geometrical optics as a really important component of optics course acquires varied teaching methods so as to have students understood the main principles. In a way the purely theoretical derivation of the optical knowledges may leave to students' lacks of intuitional comprehension of the discipline. We explore more efficiently and adaptively teaching ways all the time to help students master the bald knowledges in optics. There are many interesting optical phenomena which appear to be inconceivable in daily life. And the phenomena were often explained incorrectly. In most cases as to classics they are related to be a spiritual phenomenon. To unscramble the strange optical phenomenon is an ideal pattern to promote the student to understand the relevant optical principles. Earlier this year we visited Sanya, 
Hainan Province, China. There is a huge statue, the South Sea Avalokitesvara, at Sanya Nanshan Buddhism Cultural Tourism Resort [1]. When we were gazing at the statue on a leaving car on gradually rising road, an unexpected visual illusion took place in which the statue seemed running after us. When we told the driver about the phenomenon, he regarded it as "Buddha apparitions". Of course, as we known, it could be explained by commonsense reasoning of geometrical optics. This instance is very interesting and can be adapted to construct an optical model in order to explain the relevant optical principle in detail.

In this paper, an optical model is developed to explain the illusion occurred on a moving object leaving in fact but approaching by environmental judgement. Such an interesting illusion analysis will play a significant role in having students understood the main principles in geometrical optics.

\section{Optical model and Analysis}

Human eye is a complex imaging system, including the cornea, anterior chamber, lens, retina et al. There is a large difference in refractive indices between the air and the cornea. The object is imaged on the retina. So the eye imaging system can be simplified into a lens with a focal length of about $20 \mathrm{~mm}$ and a retina structure. The optical phenomena mentioned earlier can be reduced to a single lens imaging process of an infinite object. The object size that human eye felling depends on the image size of the object project on the retina. The height of the statue is about 108 meters and far away from us, the coconut trees near us as the reference substance with a height of 10 meters. The positions of the statue and the coconut trees are shown in Fig.1. 


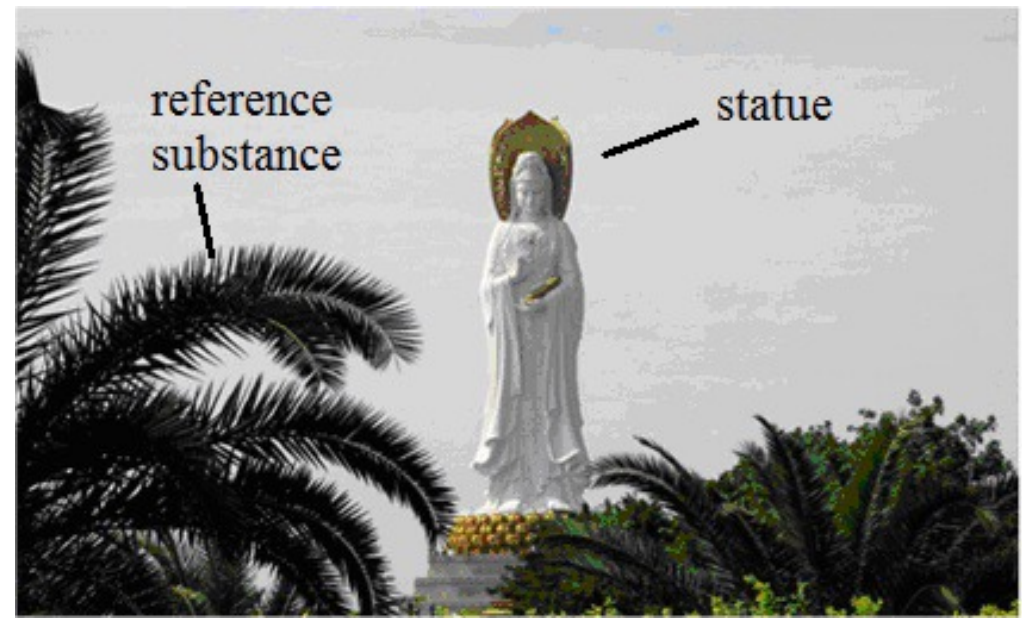

Fig. 1 sketch of the positions of the statue and the coconut trees

The corresponding imaging optical paths are shown in Fig.2.

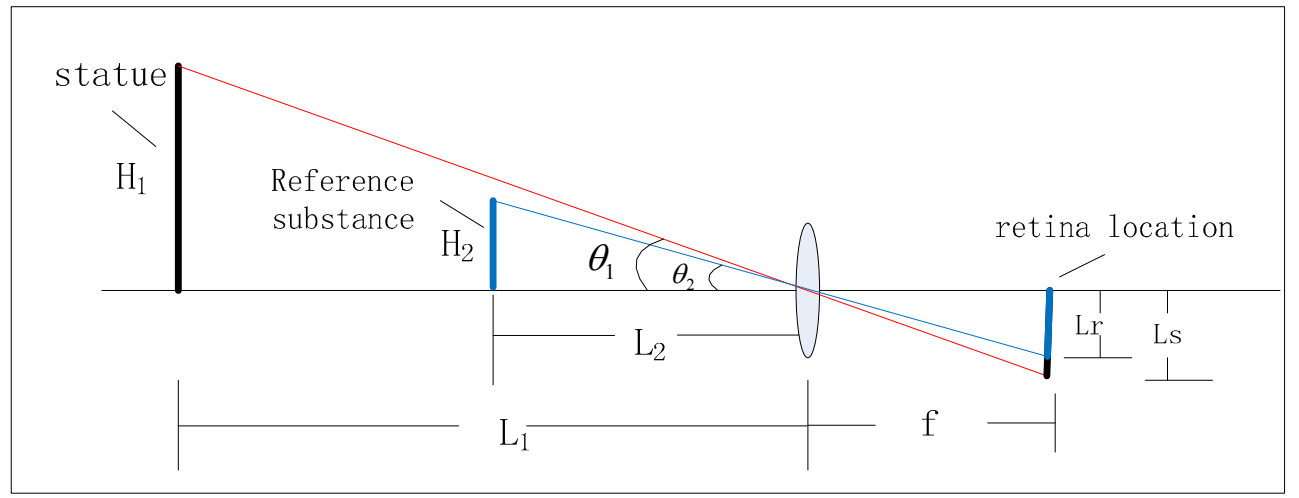

Fig.2.imaging optical path, $H_{1}$ :height of statue, $H_{2}$ :height of reference substance, $L_{S}$ :image

height of statue, $L_{r}$ : image height of reference substance.

From the geometric relations we can obtain:

$$
\operatorname{tg} \theta_{1}=\frac{H_{1}}{L_{1}} \quad \operatorname{tg} \theta_{2}=\frac{H_{2}}{L_{2}}
$$

The images size can be written as

$$
L_{s}=f \bullet \frac{H_{1}}{L_{1}} \quad L_{r}^{\prime}=f \bullet \frac{H_{2}}{L_{2}}
$$

When the car leaving, the observation distance increase $\Delta L$. The new imaging optical path of the statue is shown in Fig.3, the image size reduced $\Delta L_{s}$. 


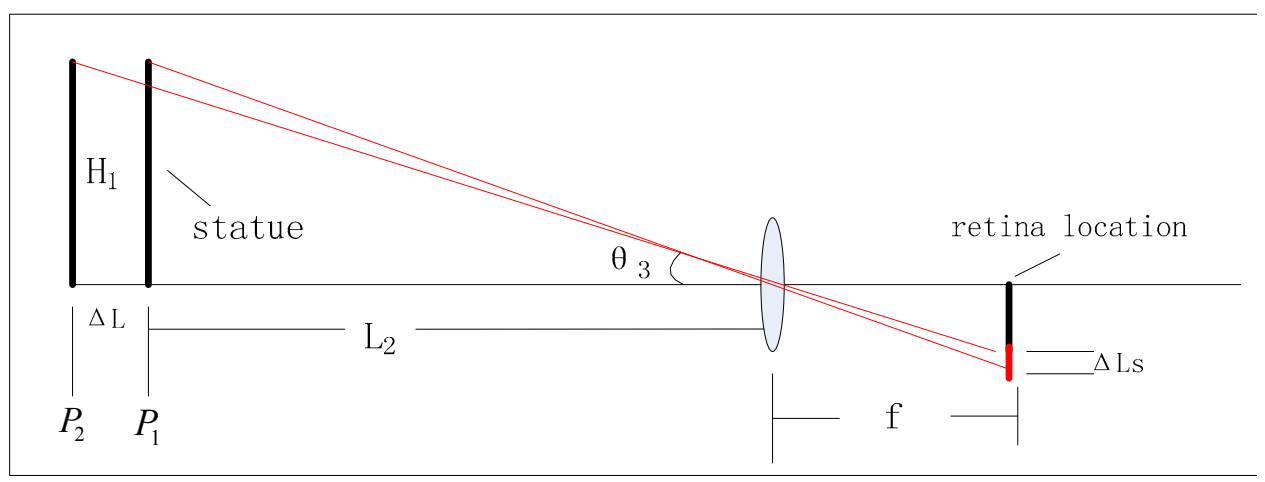

Fig.3. imaging optical path of the statue when car leaving. $P_{1}$ : The beginning statue position, $P_{2}$ :

the statue position after car moved

$$
\operatorname{tg} \theta_{3}=\frac{H_{1}}{L_{1}+\Delta L}
$$

The images size can be written as

$$
L_{s}^{\prime}=f \bullet \frac{H_{1}}{L_{1}+\Delta L}
$$

The image size reduced proportion of the statue is obtained:

$$
\frac{\Delta L_{s}}{L_{s}}=\frac{L_{s}-L_{s}^{\prime}}{L_{s}}=\frac{f \bullet H_{1} / L_{1}-f \bullet \frac{H_{1}}{L_{1}+\Delta L}}{f \bullet \frac{H_{1}}{L_{1}}}=\frac{\Delta L}{L_{1}+\Delta L}
$$

At the same time, the reference substance imaging optical path is shown in Fig.4:

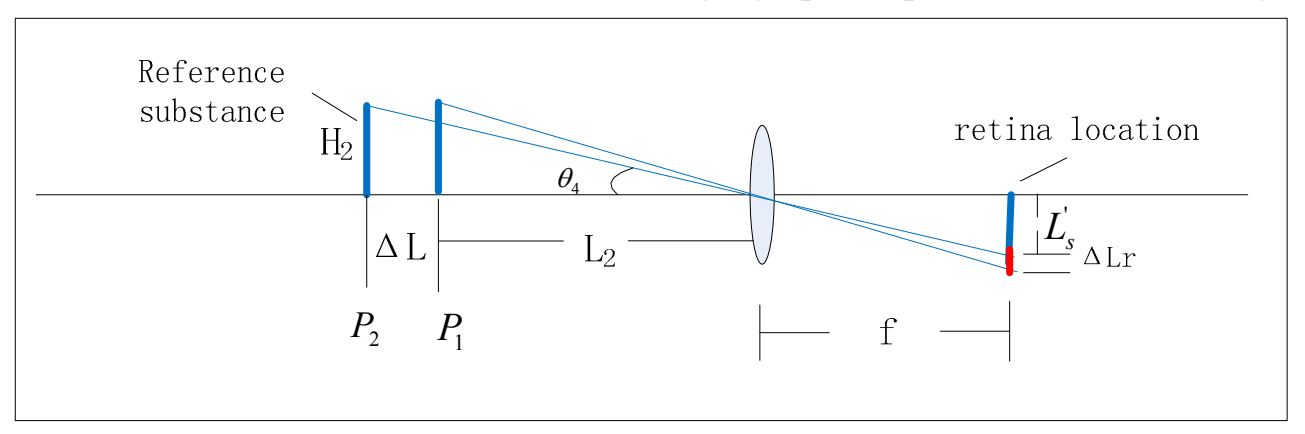

Fig.4. imaging optical path of the reference when car leaving, $P_{1}$ : The beginning reference substance position, $P_{2}$ : the reference substance position after car moved

$$
\operatorname{tg} \theta_{4}=\frac{H_{2}}{L_{2}+\Delta L}
$$

The images size can be written as 


$$
L_{r}^{\prime}=f \cdot \frac{H_{2}}{L_{2}+\Delta L}
$$

The image size reduced proportion of the reference substance is obtained:

$$
\frac{\Delta L_{r}}{L_{r}}=\frac{L_{r}-L_{r}}{L_{s}}=\frac{f \bullet H_{2} / L_{2}-f \bullet \frac{H_{2}}{L_{2}+\Delta L}}{f \bullet \frac{H_{2}}{L_{2}}}=\frac{\Delta L}{L_{2}+\Delta L}
$$

Because the statue is far more than reference substance from us, so

$$
L_{1}+\Delta L>>L_{2}+\Delta L
$$

Then according to equations (5) and (8), we find

$$
\frac{\Delta L_{s}}{L_{s}}<\frac{\Delta L_{r}}{L_{r}}
$$

The image sizes decrease process can be shown in Fig.5. When car leaving the same distance, the image size reduced proportion of the statue and reference substance is different. The decrease proportion of objects image in the remote distance is smaller than that of the near object.

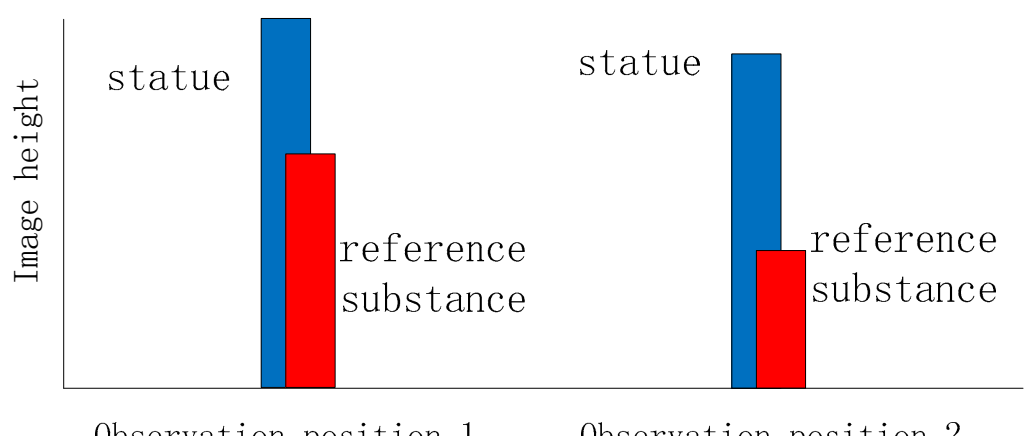

Fig.5. the image size in different observation position

The actual image obtained by eyes during the process could be shown in Fig.6. 


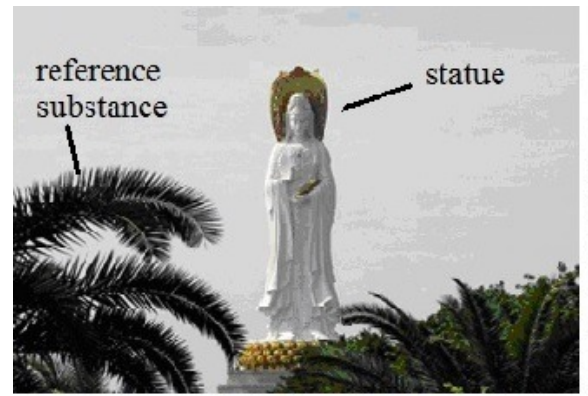

(a)

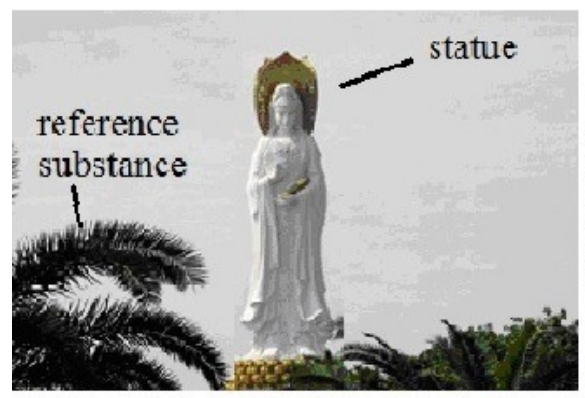

(c)

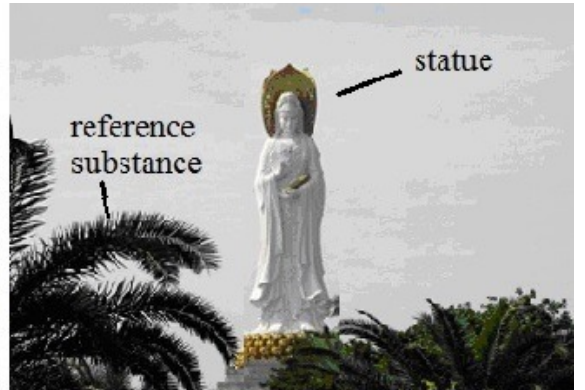

(b)

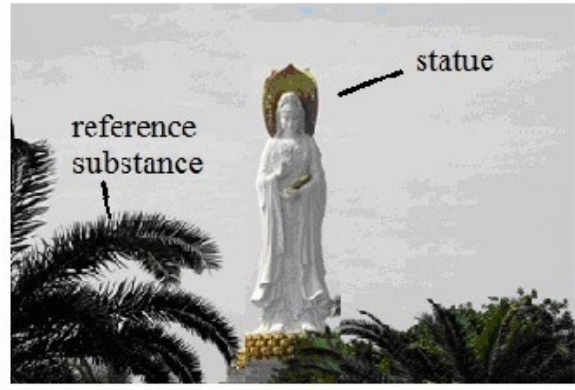

(b)

Fig.6 images on the retina at different observation position. (a) image obtained before car moving

(b) image after car moving a distance (c) proportional amplification image of (b)

When car leaving the same distance, the statue and coconut tree images on retina are become smaller shown as Fig. (b) compare to Fig.6 (a). Since the two objects in the same field of view, the coconut trees in the vicinity narrowed quickly than the statue far away when the car moving. So the statue seems to be getting bigger in the tourist brain due to the contrast of statue and coconut tree image. When the statue seems bigger showed in Fig.6(c) which is proportional amplification image of Fig. (b), the statue looks as following the leaving tourist compares to Fig.6 (a).

Obviously, the visual illusion is caused by the different change speeds of the two object images sizes. But difference of the speed need large enough and the observation object must huge enough can only cause visual illusion. The heights and positions of the statue and reference substance meet these conditions in our example. So the unexpected visual illusion took place in which the statue seemed running after us.

\section{Summary}

We developed an optical model to explain the unexpected visual illusion, demonstrated that phenomenon in fact was caused in image sizes comparison by observation environment. It a really good example to have students understood the 
main principles in geometrical optics. There are many similar cases could be introduced into classroom teaching in near future.

\section{References}

[1] http://www.nanshan.org.cn/guanyin/index.html 